\title{
Co-orbital restricted three-body problem and its application
}

\author{
LI GuangYu
}

The Purple Mountain Observatory of the Chinese Academy of Sciences and the Department of Astronomy, Nanjing University, cooperating with the Albert Einstein Institute in Germany, put forward a model of the planar co-orbital circular restricted three-body problem. We supplied the equations of motion, a set of approximation formulas, and an approximate semi-analytical solution. This significant study is reported in Vol. 53, No. 1 of SCIENCE CHINA Physics, Mechanics \& Astronomy.

As a branch of the famous three-body problem in the field of celestial mechanics, the co-orbital motion of natural bodies was recognized by astronomers a long time ago. In 1918, the Japanese astronomer T. Hirayama found that the orbital elements of some asteroids were close to each other. He proposed that asteroids with three orbital elements (the semi- major axis $a$, eccentricity $e$, and orbital inclination $i$ ) that are close to each other should be called co-orbital. The most well-known cases of co-orbits in natural objects are the Trojan groups of asteroids, of which the number of registered discovered asteroids already exceeds 2900. Co-orbital phenomena also exist in the motion of stars in the same spiral arm of the Milky Way galaxy, and in the motion of natural and man-made satellites.

The planned gravitational wave observatory LISA is regarded as an approximate example of the co-orbital circular restricted three-body problem. A rough method, proposed by the authors, has been used to design and optimize the LISA orbit with 10 years of designed life.

After further analysis and study, the authors greatly improved their previous method. The new method has extended the applicable region from 10 years to over 1000 years for most of the range of the initial values. As a typical example, they found that the barycenter of the LISA constellation moves in a horseshoe-shaped orbit around the Sun near the Earth's orbit for a period of 468 years: first, along the neighboring outer circumference of the horse-shoe-shaped orbit for 233.7 years; then, in the neighborhood along the inner circumference of the horse-shoe-shape for 234.4 years.

The authors believe their work is a new development in the field of the restricted three-body problem. The method will be extended to study the stability of this kind of orbit and the effect of orbital eccentricity of planets, the exploration of a more accurate analytical solution, the study of the orbits of the Trojan asteroids, near-earth asteroids, in the design of spacecraft (which are approximately co-orbital), and the study of the motion of co-orbital stellar families in the Milky Way galaxy.

The Purple Mountain Observatory is a significant research institute in the field of celestial mechanics. The group, headed by Prof. LI GuangYu, cooperating with Prof. YI ZhaoHua in Nanjing University and Researcher Gerhard HEINZEL in AEI, Germany, has made great strides in the fields of NEO exploration, planetary and lunar ephemeris, orbit design, and optimization of planetary spacecraft.

Funding from the National Natural Science Foundation of China (Grant Nos. 10503013, 10933004), and the Foundation of Minor Planets of Purple Mountain Observatory supported this research.

See the article: Yi Z H, Li G Y, Heinzel G, et al. The co-orbital restricted three-body problem and its application. Sci China Phys Mech Astron, 2010, 53: $171-178$ 